\title{
Interdigitating Dendritic Cell Sarcoma
}

\author{
Venkata K. Pokuri, MDª; Mihai Merzianu, MD ; Shipra Gandhi, MDc; Junaid Baqai, MD; \\ Thom R. Loree, $\mathrm{MD}^{\mathrm{d}}$; and Seema Bhat, $\mathrm{MD}^{\mathrm{a}}$
}

\begin{abstract}
Interdigitating dendritic cell sarcoma (IDCS) is an extremely rare dendritic cell tumor with slightly more than 100 cases reported in the English literature. This report discusses a case of localized IDCS involving cervical lymph nodes and provides a literature review of clinicopathologic aspects and treatment outcomes. (J Natl Compr Canc Netw 2015;13:128-132)
\end{abstract}

\section{NCCN: Continuing Education}

\section{Accreditation Statement}

This activity has been designated to meet the educational needs of physicians and nurses involved in the management of patients with cancer. There is no fee for this article. No commercial support was received for this article. The National Comprehensive Cancer Network (NCCN) is accredited by the ACCME to provide continuing medical education for physicians.

NCCN designates this journal-based CME activity for a maximum of 1.0 AMA PRA Category 1 Credit(s) ${ }^{\mathrm{TM}}$. Physicians should claim only the credit commensurate with the extent of their participation in the activity.

From the a Department of Medical Oncology and 'Department of Pathology, Roswell Park Cancer Institute; 'Department of Medicine, State University of New York; and ${ }^{\mathrm{d} D e p a r t m e n t}$ of Head \& Neck and Plastic \& Reconstructive Surgery, Erie County Medical Center, Buffalo, New York.

Submitted January 29, 2014; accepted for publication June 5, 2014

The authors have disclosed that they have no financial interests, arrangements, affiliations, or commercial interests with the manufacturers of any products discussed in this article or their competitors.

Correspondence: Seema Bhat, MD, Department of Medical Oncology,

Roswell Park Cancer Institute, Elm \& Carlton Streets, Buffalo, NY 14263.

E-mail: Seema.Bhat2@RoswellPark.org

\section{EDITOR}

Kerrin M. Green, MA, Assistant Managing Editor, JNCCN-Journal of the National Comprehensive Cancer Network

Ms. Green has disclosed that she has no relevant financial relationships.
NCCN is accredited as a provider of continuing nursing education by the American Nurses Credentialing Center's Commission on Accreditation.

This activity is accredited for 1.0 contact hours. Accreditation as a provider refers to recognition of educational activities only; accredited status does not imply endorsement by NCCN or ANCC of any commercial products discussed/displayed in conjunction with the educational activity. Kristina M. Gregory, RN, MSN, OCN, is our nurse planner for this educational activity.

All clinicians completing this activity will be issued a certificate of participation. To participate in this journal CE activity: 1) review the learning objectives and author disclosures; 2 ) study the education content; 3) take the posttest with a $66 \%$ minimum passing score and complete the evaluation at http://education.nccn.org/ node/60900; and 4) view/print certificate.

Release date: February 18, 2015; Expiration date: February 18, 2016

\section{Learning Objectives}

Upon completion of this activity, participants will be able to:

- Evaluate the patient demographics, clinicopathologic features, and therapeutic outcomes of documented IDCS cases

- Discuss challenges in the accurate pathologic diagnosis, staging, and treatment of patients with IDCS

\section{CE AUTHORS}

Deborah J. Moonan, RN, BSN, Director, Continuing Education, has disclosed that she has no relevant financial relationships.

Ann Gianola, MA, Manager, Continuing Education Accreditation \& Program Operations, has disclosed that she has no relevant financial relationships.

Kristina M. Gregory, RN, MSN, OCN, Vice President, Clinical Information Operations, has disclosed that she has no relevant financial relationships. Rashmi Kumar, PhD, Senior Manager, Clinical Content, has disclosed that she has no relevant financial relationships.

Hema Sundar, PhD, Oncology Scientist/Senior Medical Writer, has disclosed that her spouse is employed by Kashiv Pharma LLC. 


\section{Case Report}

An 81-year-old Caucasian man presented with a 4-month history of a gradually enlarging and painless left neck mass. He had no systemic or constitutional symptoms. His medical history included poorly differentiated squamous cell carcinoma of the scalp, which was treated with wide local excision and skin graft 2 years prior to presentation, and high-grade papillary urothelial carcinoma of the bladder without muscle invasion 1 year prior, which was treated with transurethral ablation. He was otherwise in good health. Physical examination revealed a $3-\mathrm{cm}$ firm, nontender, immobile mass in the left posterior triangle of the neck. No axillary, supraclavicular, or inguinal lymphadenopathy was present, and no organomegaly was identified. CBC count and results of renal and liver function tests were within normal limits. Results of the HIV antibody test were negative. An excisional biopsy of the neck mass revealed interdigitating dendritic cell sarcoma (IDCS) with lymph node involvement. A PET scan performed a month after the excision revealed persistent fluorodeoxyglucose (FDG) avidity in an area of the left posterior triangle of the neck measuring $1.08 \mathrm{~cm}$ in maximum transverse diameter, with a maximum standardized uptake value of 3.14 (Figure 1). The presence of residual tumor was the likely cause of persistent FDG avidity at the surgical site; local radiation or reexcision was recommended. A left modified radical neck dissection was performed to exclude a metastasis from prior squamous cell carcinoma of the scalp; 53 lymph nodes were removed, 1 of which showed IDCS and the remainder were benign. The patient was offered local radiation postsurgery, but

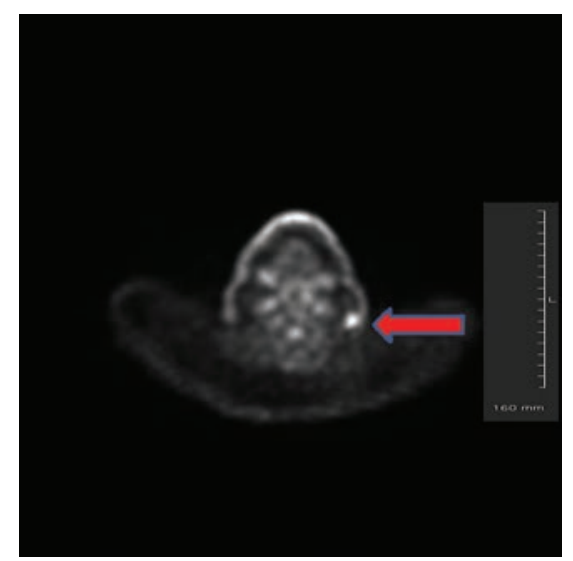

Figure 1 PET scan revealing an FDG-avid residual lymph node in the left posterior triangle of the neck at the previous surgical site (arrow). refused adjuvant therapy. The patient has been disease-free for more than 18 months.

\section{Pathology}

Histologic sections of the lymph node showed diffuse replacement of the normal architecture by a spindle cell neoplasm, with effacement of subcapsular sinuses and extension into perinodal fat (Figure 2A/B). Neoplastic cells were disposed in fascicles with a focal storiform pattern, had mostly large spindle-shaped vesicular nuclei, occasional prominent nucleoli, and abundant eosinophilic cytoplasm with well-defined cellular borders (Figure 2C). In other areas, tumor cells had epithelioid or histiocytoid cell morphology or had convolutions resembling Langerhans cells. Occasional bizarre, binucleated, or multinucleated (some Langhanslike) forms were present in addition to a few cells with hyperchromatic nuclei. Rare bilobed nuclei resembling Reed-Sternberg cells were seen (Figure 2D). Mitotic activity was increased (29 mitoses per 50 high-power field) with many atypical mitoses and few areas of tumor necrosis.

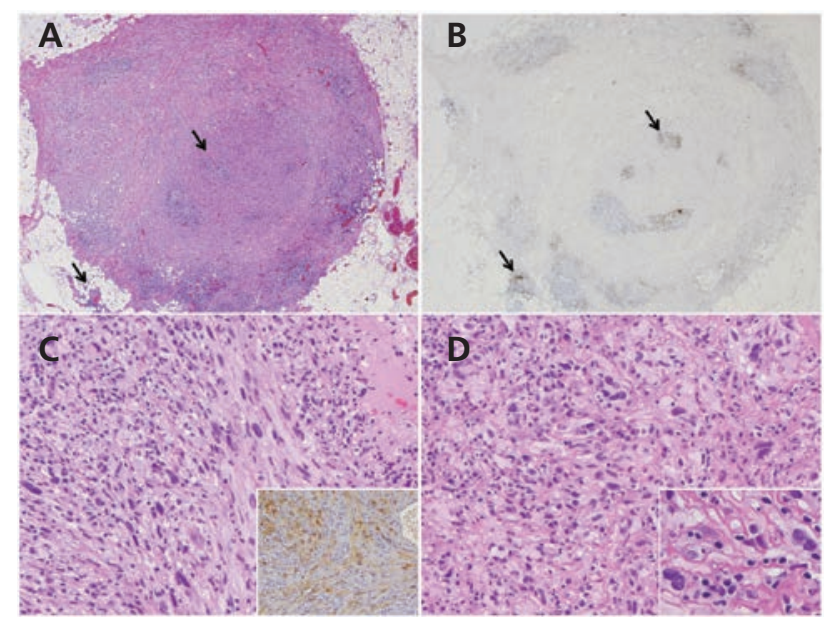

Figure 2 (A) Lymph node with paracortical and interfollicular involvement by interdigitating dendritic cell sarcoma, with effacement of normal architecture and extension into adjacent adipose tissue (hematoxylin-eosin, original magnification $\times 20$ ). Occasional residual disrupted cells are seen (arrows). (B) The disrupted follicles are highlighted by CD21, not expressed in the tumor cells (CD21, original magnification $\mathrm{x} 20$ ). (C) The neoplastic cells have variable size and predominantly spindle cell morphology, and show nuclear and cytoplasmic $\$ 100$ labeling (inset: S100; original magnification $\times 200$ ). Note the intermixed small lymphocytes and plasma cells in the background. (D) Neoplastic cells are predominantly epithelioid; some histiocytoid or Langerhans cell-like and rare multinucleated forms are seen in a hyalinized background (hematoxylin-eosin, original magnification x200). Rare cells can superficially mimic Reed-Sternberg cells or variants in the sclerotic background (inset; hematoxylin-eosin, original magnification $\mathrm{x600)}$ ). 
Immunohistochemical studies showed that neoplastic cells strongly and diffusely expressed vimentin, S100 (cytoplasmic and nuclear pattern; Figure 2C), and $\mathrm{p} 53$, and were negative for CD1a, CD3, CD4, CD15, CD20, CD21, CD23, CD30, CD34, CD35, CD43, CD45, CD68, CD163, lysozyme, pancytokeratin, CK 5/6, EMA, p63, HMB-45, Melan-A, D2-40, SMA, and desmin. The proliferation index determined with $\mathrm{Ki}-67$ labeling averaged 20\% (range, $10 \%-40 \%)$. Background lymphocytes were mostly of T-cell lineage, and residual B cells were sparse.

Results of in situ hybridization for Epstein-Barr virus-encoded small RNA were negative, and Band T-cell polymerase chain reaction clonality studies were polyclonal. The previous squamous cell carcinoma of the scalp was histologically different from the nodal tumor at review.

\section{Discussion}

Dendritic cells (DCs) are a heterogenous group of nonlymphoid, nonphagocytic immune accessory cells present in lymphoid and nonlymphoid organs. They are key antigen-presenting cells and initiators of the immune response. Four types of DCs exist in lymph nodes: follicular, interdigitating, Langerhans, and fibroblastic cells. ${ }^{1}$ DC neoplasms are rare and represent less than $1 \%$ of all lymph node tumors. ${ }^{2}$ These tumors were previously classified as lymphomas, sarcomas, or histiocytic neoplasms on histologic grounds alone, but recent diagnostic advancements have refined their classification. The WHO currently classifies these neoplasms based on their putative histogenesis and phenotype into myeloidderived histiocytic neoplasms (histiocytic sarcoma), myeloid-derived dendritic neoplasms (Langerhans cell histiocytosis and sarcoma, IDCS, indeterminate DC tumor, and probably plasmacytoid DC tumor), and stromal-derived dendritic neoplasms (follicular DC sarcoma and fibroblastic reticular cell tumor). ${ }^{3}$

Interdigitating DCs (IDC) present antigens to $\mathrm{T}$ cells and are normally seen in T-cell zones of lymphoid organs, such as the paracortex of lymph nodes and the periarteriolar lymphoid sheaths in the spleen. ${ }^{4}$ IDCs are believed to originate from differentiation of marrow precursor cells; Langerhans cells can mature into IDC upon migration to lymph nodes, thus enhancing their antigen-presenting function. ${ }^{5-7}$
IDCS is a rare neoplasm. A recent comprehensive review of dendritic sarcomas by Saygin et $\mathrm{al}^{8}$ presented the demographics, clinicopathologic features, and therapeutic outcomes of 100 cases of IDCS. Patients are generally adult men with a median age at diagnosis of 56.5 years. ${ }^{8}$ Saygin et $\mathrm{al}^{8}$ reported that, of 39 patients, 16 (41\%) were Asian, 19 (49\%) were Caucasian, 2 (5\%) were Hispanic, and 2 (5\%) were African American. The most common presentation was painless localized lymphadenopathy, occurring in $47 \%$ of cases (sites in order of frequency: cervical, axillary, abdominal, inguinal, or mediastinal regions), ${ }^{8}$ and $25 \%$ of patients presented with isolated extranodal disease (the most common sites being liver, lung, spleen, bone marrow, and gastrointestinal tract); the remaining patients presented with both nodal and extranodal disease. ${ }^{8}$ Systemic symptoms (fever, night sweats, weight loss, and fatigue) were uncommon and mostly occurred in patients with both nodal and extranodal disease. ${ }^{8}$ The incidence of distant metastasis was $39 \%$, with the most common sites of metastasis being lymph nodes (29\%), lung (11\%), liver (11\%), and bone marrow $(8 \%) .8$

The etiopathogenesis of IDCS is unknown. Epstein-Barr virus has been suggested as a causative factor in the pathogenesis of follicular DC sarcomas, ${ }^{9}$ but not in IDCS. In addition, human herpesvirus- 8 genome has not been identified in IDCS. ${ }^{10}$ However, the association of IDCS with preceding or concomitant malignant neoplasms has been suggested in several case reports. Saygin et $\mathrm{al}^{8}$ reported occurrence rates of $12 \%$ and $9 \%$ in hematopoietic (eg, T-cell lymphoblastic lymphoma, low-grade follicular lymphoma, chronic lymphocytic leukemia/small lymphocytic lymphoid tumor [CLL/SLL], and large B-cell lymphoma) and solid organ malignancies (eg, liver, stomach, colon, brain, breast, and skin neoplasms), respectively, in patients with IDCS during their lifetime. This high frequency of concomitant malignancies may be coincidental and related to aging, or may support the hypothesis that DCs introduce tumor antigens to immune cells and that their dysfunction could result in a diminished response to the neoplasm. In the present case, the patient had a history of 2 solid organ malignancies (bladder and skin).

Previous case reports and small series have demonstrated a clonal relationship between low-grade B-cell lymphomas (follicular lymphoma and CLL/ SLL) and histiocytic/DC tumors through identical 
molecular alterations and monoclonal immunoglobulin heavy chain gene rearrangements. ${ }^{11-13}$ This finding of shared underlying molecular alterations in low-grade B-cell lymphomas and IDCS led to the concept of transdifferentiation of B-cell neoplastic cells into mature neoplastic DC, ${ }^{11,13}$ of which IDCS is common. ${ }^{12}$

A diagnosis of IDCS can be challenging to a general pathologist and even a hematopathologist because of its rarity and morphologic similarity to a variety of primary and metastatic spindle cell neoplasms involving lymph nodes. Because of the morphologic overlap of histiocytic, Langerhans, follicular, reticular, and interdigitating tumors, ancillary studies are needed for a definitive diagnosis. Other tumors that require exclusion are spindle cell carcinoma, melanoma, and a variety of mesenchymal neoplasms. Approximately $11 \%$ of IDCS cases are misdiagnosed as lymphoma, melanoma, peripheral nerve sheath tumor, or malignant fibrous histiocytoma. ${ }^{8}$ Histologically, IDCS shows a diffuse proliferation of round-ovoid cells to spindle cells, with a variety of growth patterns, including sinusoidal, nesting, fascicular, and storiform, ${ }^{14-16}$ such as in the present case, and the proliferation can partially or completely replace the affected tissue. A collagenous or hyalinized background, with increased reticulin fibers surrounding individual cells, may be present. ${ }^{17,18}$ Microscopically, individual cells have a slender, spindled to plump (histiocytoid) appearance with ill-defined cell borders, abundant eosinophilic cytoplasm, and enlarged indented nuclei. The cells resemble Langerhans cells in microscopic appearance, but lack both Birbeck granules ultrastructurally,14-16 and expression of CD1a and langerin. Occasional epithelioid cells, bizarre atypia, or Reed-Sternberg-like cells, as seen in the present case, may superficially resemble classical Hodgkin lymphoma. Necrosis is unusual $^{19}$ and the mitotic rate is variable. Tumor cells are positive for S-100, vimentin, HLA-DR, and CD68, with CD68 weakly and variably expressed. In the present case, all histiocytic markers were negative. Variable staining has been reported for CD1a, CD11c, CD45RB, CD45RO, CD4, and CD14. ${ }^{2,20}$ IDCS is negative for CD21, CD35, CD3, and CD20. The Ki-67 labeling index usually ranges between $10 \%$ to $20 \% .{ }^{21}$ IDCS may be difficult to distinguish from metastatic melanoma, which can have a similar immunohistochemical profile (S-100+, CD68+/-). ${ }^{22}$ The lack of other melanocytic markers establishes the diagnosis.
The significant immunophenotype variability of DCs may reflect the multiple pathways postulated in normal DC development. ${ }^{4-7}$

After establishing the pathologic diagnosis, staging should be completed, which includes imaging with CT scans and a bone marrow biopsy to evaluate for systemic disease. Primary bone marrow involvement seems to be less common in adults $(11 \%$ in Saygin et $\left.\mathrm{al}^{8}\right)$. Whether bone marrow biopsy is required in patients with localized disease who lack cytopenias and/or systemic symptoms is unclear. The role of PET scans in these rare tumors is also unclear. IDCS has been shown to be FDG-avid in some cases, ${ }^{23}$ and PET can be used to monitor treatment response. ${ }^{24,25}$ In the present case, PET scan detected residual disease and prompted surgical reintervention.

Currently, no standard of care exists for the treatment of IDCS. Localized disease is managed with surgery or radiation. Saygin et $\mathrm{al}^{8}$ reported no significant difference in survival among patients with localized IDCS who underwent surgery compared with those treated with nonsurgical modalities. Therefore, radiotherapy can be a reasonable alternative to surgery in select cases. However, the role of adjuvant radiotherapy after surgery is unclear. Chemotherapy is the preferred treatment for patients with metastatic IDCS. Combination chemotherapy regimens administered for aggressive lymphomas can be considered, such as CHOP (cyclophosphamide, doxorubicin, vincristine, prednisone), ABVD (doxorubicin, bleomycin, vinblastine, dacarbazine), and ICE (ifosfamide, carboplatin, etoposide). ${ }^{8} \mathrm{~Pa}$ tients with localized disease had significantly superior overall survival compared with those with metastatic disease (2-year survival rates, $68.1 \%$ vs $15.8 \%$, respectively), compared with follicular dendritic cell sarcoma as stage did not significantly alter overall survival. ${ }^{8}$ The patient in the present case presented with localized IDCS that was treated with surgery alone and has been disease-free for more than 18 months. Young age ( $\leq 40$ years), presence of intraabdominal involvement, and combined nodal and extranodal involvement were associated with adverse outcomes (local recurrence, distant metastasis, or death), whereas gender, tumor size $(\geq 6 \mathrm{~cm})$, and histologic features such as presence of necrosis, high mitotic rate, and lymphoplasmacytic infiltration did not seem to predict recurrence or survival. ${ }^{8}$ 
Pokuri et al

\section{Conclusions}

IDCS is an extremely rare tumor, with slightly more than 100 cases reported to date. The disease can be associated with other various malignancies (including hematolymphoid and solid tumors) occurring either synchronously or metachronously, which may create challenges for disease management. The histomorphology and immunoprofile help differentiate IDCS from other spindle cell neoplasms and metastatic melanomas. Patients with localized IDCS are treated similarly to patients with soft tissue sarcoma with primary surgical resection, and have better outcomes compared with those with metastatic disease. Young age, intra-abdominal involvement, and advanced stage are poor predictors of outcome. The role of adjuvant therapy remains unclear. Chemotherapy options for metastatic disease are controversial.

\section{References}

1. Fonseca R, Yamakawa M, Nakamura S, et al. Follicular dendritic cell sarcoma and interdigitating reticulum cell sarcoma: a review. Am J Hematol 1998;59:161-167.

2. Pileri SA, Grogan TM, Harris NL, et al. Tumours of histiocytes and accessory dendritic cells: an immunohistochemical approach to classification from the International Lymphoma Study Group based on 61 cases. Histopathology 2002;41:1-29.

3. Jaffe R, Pileri SA, Facchetti F, et al. Histiocytic and dendritic cell neoplasms. In: Swerdlow SH, Campo E, Harris NL, et al, eds. WHO Classification of Tumours of Haematopoietic and Lymphoid Tissues. 4th ed. Lyon, France: IARC Press; 2008:353-367.

4. Steinman RM, Pack M, Inaba K. Dendritic cells in the T-cell areas of lymphoid organs. Immunol Rev 1997;156:25-37.

5. Wood GS, Turner RR, Shiurba RA, et al. Human dendritic cells and macrophages. In situ immunophenotypic definition of subsets that exhibit specific morphologic and microenvironmental characteristics. Am J Pathol 1985; 119:73-82.

6. Rosenzwajg M, Canque B, Gluckman JC. Human dendritic cell differentiation pathway from CD34+ hematopoietic precursor cells. Blood 1996;87:535-544.

7. Wu L, Liu YJ. Development of dendritic-cell lineages. Immunity 2007;26:741-750.

\section{Instructions for Completion}

To participate in this journal CE activity: 1) review the learning objectives and author disclosures; 2 ) study the education content; 3 ) take the posttest with a $66 \%$ minimum passing score and complete the evaluation at http://education.nccn.org/ node/60900; and 4) view/print certificate. After reading the article, you should be able to answer the following multiple-
8. Saygin C, Uzunaslan D, Ozguroglu M, et al. Dendritic cell sarcoma: a pooled analysis including 462 cases with presentation of our case series. Crit Rev Oncol Hematol 2013;88:253-271.

9. Choe JY, Go H, Jeon YK, et al. Inflammatory pseudotumor-like follicular dendritic cell sarcoma of the spleen: a report of six cases with increased IgG4-positive plasma cells. Pathol Int 2013;63:245-251.

10. Barwell $\mathrm{N}$, Howatson $\mathrm{R}$, Jackson $\mathrm{R}$, et al. Interdigitating dendritic cell sarcoma of salivary gland associated lymphoid tissue not associated with HIV-8 or EBV infection. J Clin Pathol 2004;57:87-89.

11. Fraser CR, Wang W, Gomez M, et al. Transformation of chronic lymphocytic leukemia/small lymphocytic lymphoma to interdigitating dendritic cell sarcoma: evidence for transdifferentiation of the lymphoma dendritic cell sarcoma: evidence for transdiffer
clone. Am J Clin Pathol 2009;132:928-939.

12. Shao $H, X i$ L, Raffeld $M$, et al. Clonally related histiocytic/dendritic cell sarcoma and chronic lymphocytic leukemia/small lymphocytic lymphoma: a study of seven cases. Mod Pathol 2011;24:1421-1432.

13. Feldman AL, Arber DA, Pittaluga $S$, et al. Clonally related follicular lymphomas and histiocytic/dendritic cell sarcomas: evidence for transdifferentiation of the follicular lymphoma clone. Blood 2008;111:5433-5439.

14. Andriko JW, Kaldjian EP, Tsokos M, et al. Reticulum cell neoplasms of lymph nodes: a clinicopathologic study of 11 cases with recognition of a new subtype derived from fibroblastic reticular cells. Am J Surg Patho 1998;22:1048-1058.

15. Weiss LM, Berry GJ, Dorfman RF, et al. Spindle cell neoplasms of lymph nodes of probable reticulum cell lineage. True reticulum cell sarcoma? Am J Surg Pathol 1990;14:405-414.

16. Luk IS, Shek TW, Tang VW, et al. Interdigitating dendritic cell tumor of the testis: a novel testicular spindle cell neoplasm. Am J Surg Pathol 1999;23:1141-1148.

17. Van Den Oord JJ, De Wolf-Peeters C, De Vos R, et al. Sarcoma arising from interdigitating reticulum cells: report of a case, studied with light and electron microscopy, and enzyme- and immunohistochemistry. and electron microscopy, and
Histopathology 1986;10:509-523.

18. Rabkin MS, Kjeldsberg CR, Hammond ME, et al. Clinical, ultrastructural immunohistochemical and DNA content analysis of lymphomas having features of interdigitating reticulum cells. Cancer 1988;61:1594-1601.

19. Gaertner EM, Tsokos M, Derringer GA, et al. Interdigitating dendritic cell sarcoma. A report of four cases and review of the literature. Am J Clin Pathol 2001;115:589-597.

20. Rousselet MC, Francois S, Croue A, et al. A lymph node interdigitating reticulum cell sarcoma. Arch Pathol Lab Med 1994;118:183-188.

21. Kapucuoglu N, Percinel S, Ventura T, et al. Dendritic cell sarcomas tumours of the breast: report of two cases. Virchows Arch 2009;454:333339.

22. Pernick NL, Dasilva M, Gangi MD, et al. "Histiocytic markers" in melanoma. Mod Pathol 1999;12:1072-1077.

23. Han SW, Kim ZS, Kim HM, et al. Interdigitating dendritic cell sarcoma occurred alone in axilla. J Korean Surg Soc 2012;82:330-334.

24. Olnes MJ, Nicol T, Duncan $M$, et al. Interdigitating dendritic cell sarcoma: a rare malignancy responsive to ABVD chemotherapy. Leuk Lymphoma 2002;43:817-821.

25. Johnson RL, Boisot S, Ball ED, Wang HY. A case of interdigitating dendritic cell sarcoma/histiocytic sarcoma - a diagnostic pitfall. Int J Clin Exp Pathol 2013;7:378-385.

\section{Posttest Questions}

1. True or False: IDCS and follicular dendritic cell sarcoma have distinct histomorphologic features but similar biology and clinical course.

2. Patients with IDCS can also have preceding or concomitant hematopoietic and solid organ malignancies. The reported occurrence rate of these malignancies during the lifetime of IDCS patients is approximately:
a. $10 \%$
b. $40 \%$

choice questions. Credit cannot be obtained for tests completed on paper. You must be a registered user on NCCN.org. If you are not registered on NCCN.org, click on "New Member? Sign up here" link on the left hand side of the Web site to register. Only one answer is correct for each question. Once you successfully answer all posttest questions you will be able to view and/or print your certificate. Software requirements: Internet.

\section{c. $70 \%$}

d. $90 \%$

3. Which of the following neoplasms should be considered in the differential diagnosis of IDCS?

a. Spindle cell carcinoma

b. Follicular dendritic cell sarcoma

c. Melanoma

d. Non-Hodgkin lymphoma

e. Langerhans cell histiocytosis

f. All of the above

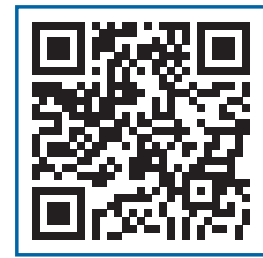

